\title{
Kernelized $\boldsymbol{k}$-Local Hyperplane Distance Nearest-Neighbor Model for Predicting Cerebrovascular Disease in Patients With End-Stage Renal Disease
}

\author{
Xiaobin Liu't, Xiran Zhang't, Yi Zhang't, Yijie Ding ${ }^{3}$, Weiwei Shan', Yiqing Huang', \\ Liang Wang ${ }^{1 *}$ and Xiaoyi Guo ${ }^{4 *}$
}

${ }^{1}$ Department of Nephrology, The Affiliated Wuxi People's Hospital of Nanjing Medical University, Wuxi, China, ${ }^{2}$ NHC Key Laboratory of Nuclear Medicine, Jiangsu Key Laboratory of Molecular Nuclear Medicine, Jiangsu Institute of Nuclear Medicine, Wuxi, China, ${ }^{3}$ Yangtze Delta Region Institute (Quzhou), University of Electronic Science and Technology of China, Quzhou, China, ${ }^{4}$ Institute of Fundamental and Frontier Sciences, University of Electronic Science and Technology of China, Chengdu, China

\section{OPEN ACCESS}

Edited by:

Yuanpeng Zhang,

Nantong University, China

Reviewed by:

Xiaoqing $\mathrm{Gu}$

Changzhou University, China

Qiusheng Shen,

Changshu Affiliated Hospital

of Nanjing University of Traditional

Chinese Medicine, China

*Correspondence:

Liang Wang

w/wxsnk@163.com

Xiaoyi Guo

kerry.guoxiaoyi@163.com

${ }^{t}$ These authors share first authorship

Specialty section:

This article was submitted to Brain Imaging Methods,

a section of the journal

Frontiers in Neuroscience

Received: 09 September 2021 Accepted: 04 October 2021

Published: 25 October 2021

Citation:

Liu X, Zhang $X$, Zhang $Y$, Ding $Y$, Shan W, Huang $Y$, Wang $L$ and Guo $X$ (2021) Kernelized k-Local Hyperplane

Distance Nearest-Neighbor Model for Predicting Cerebrovascular Disease in Patients With End-Stage

Renal Disease.

Front. Neurosci. 15:773208. doi: 10.3389/fnins.2021.773208
Detecting and treating cerebrovascular diseases are essential for the survival of patients with chronic kidney disease (CKD). Machine learning algorithms can be used to effectively predict stroke risk in patients with end-stage renal disease (ESRD). An imbalance in the amount of collected data associated with different risk levels can influence the classification task. Therefore, we propose the use of a kernelized $k$-local hyperplane nearest-neighbor model (KHKNN) for the effective prediction of stroke risk in patients with ESRD. We compared our proposed method with other conventional machine learning methods, which revealed that our method could effectively perform the task of classifying stroke risk.

Keywords: cerebrovascular disease, end-stage renal disease, local hyperplane, klotho, FGF23

\section{INTRODUCTION}

Chronic kidney disease (CKD) has become a prominent disease affecting global health. According to existing research, the global incidence of CKD is approximately $8-16 \%$ and has been increasing yearly (Jha et al., 2013). Cerebrovascular diseases, such as stroke, represent major CKD complications that lead to neurological dysfunction and death, with negative impacts on prognosis in patients with CKD. Cerebral apoplexy, which is a primary cause of death among patients with CKD, refers to a series of adverse events, including cerebral ischemia, hypoxia, and cerebral dysfunction, caused by acute cerebral vascular rupture or acute cerebrovascular embolism (Kelly and Rothwell, 2020). A cohort study showed that CKD progression and a decline in the glomerular filtration rate increased the stroke risk among patients with CKD by nearly $40 \%$, accompanied by a significant increase in the mortality rate (Toyoda and Ninomiya, 2014). Therefore, exploring the risk factors associated with stroke among the CKD population and identifying effective early interventions are necessary steps to reducing morbidity and mortality due to stroke.

Hypertension, diabetes, and dyslipidemia are traditional risk factors that contribute to the development of cerebrovascular diseases in patients with CKD. In addition, recent studies have revealed non-traditional risk factors, such as inflammation, oxidative stress, and CKD-mineral bone disease (CKD-MBD), that impact the occurrence and development of cerebrovascular diseases among patients with CKD. These non-traditional risk factors accelerate a series of 
pathological processes, such as cerebrovascular endothelial injury and sclerosis, in patients with CKD, leading to cerebrovascular calcification, further changing hemodynamics, and ultimately causing cerebrovascular events (Allen and Bayraktutan, 2009).

Our previous work identified abnormal FGF23 and Klotho levels, inflammatory status, and malnutrition were the unconventional risk factors for vascular calcification and CKD-MBD in patients with end-stage renal failure (Maraj et al., 2018). Using machine learning methods to analyze the risk factors of CKD-MBD in patients with end-stage renal failure, we have found that elevated serum FGF23 levels in patients with ESRD is an independent risk factor for abdominal aortic calcification (Liu et al., 2021). Recent studies have also identified FGF23 as an independent risk factor for cerebrovascular diseases in both CKD and non-CKD populations (Wright et al., 2016). The CHADS2 (congestive heart failure, hypertension, age $=75$ years, diabetes mellitus, stroke) and CHA2DS2-VASc (congestive heart failure, hypertension, age $\geq 75$ years, diabetes mellitus, stroke or transient ischemic attack vascular disease, age 65 to 74 years, sex category) scores are currently well-recognized methods for predicting the risk of stroke in patients with CKD (Hsu et al., 2020). Therefore, in this study, based on previous research findings, we used machine learning algorithms to develop models that explore the scientificity and veracity of both traditional and non-traditional risk factors combined with the CHADS2 stroke scoring tool and an abdominal aortic calcification scoring method for the prediction of stroke risk in patients with ESRD, which could help clinicians identify cerebrovascular disease and provide early interventions by assessing various risk factors, potentially delaying the occurrence and development of stroke, reducing morbidity and mortality, and improving prognosis among patients with ESRD.

\section{MATERIALS AND METHODS}

\section{Assessment of the CHADS2 and CHA2DS2-VASc Scores}

We calculated the CHADS2 score based on the scoring system, as follows (de Bie et al., 2017): 1 point each was assigned for age $\geq 75$ years, the presence of hypertension, diabetes mellitus, and congestive heart failure, and 2 points each were assigned for transient ischemic attack or a history of stroke. In addition, we calculated the CHA2DS2-VASc score based on the scoring system, as follows: 1 point each was assigned for congestive heart failure, hypertension, age between 65 and 74 years, diabetes mellitus, female sex, and vascular disease, whereas 2 points each were assigned for a history of stroke and age $\geq 75$ years. CKD was defined as estimated glomerular filtration rate (eGFR) $<60 \mathrm{~mL} / \mathrm{min} / \mathrm{m}^{2}$ and classified as stages 3,4 , or 5 based on the eGFR level $(30-59,15-29$, or $<15 \mathrm{~mL} / \mathrm{min} / 1.73$ $\mathrm{m}^{2}$, respectively) combined with kidney damage lasting for longer than 3 months. Patients were categorized into two groups according to their CHADS2 and CHA2DS2-VASc risk scores: (1) low-risk group (0-1 score) for CHADS2 and CHA2DS2-VASc scores and (2) high-risk group ( $\geq 2$ scores) for CHADS2 and CHA2DS2-VASc scores.

\section{Calculation of the Nutritional Indexes}

The Geriatric Nutrition Risk Index $($ GNRI $)=[14.89 \times$ serum albumin $(\mathrm{g} / \mathrm{dl})]+[41.7 \times$ (actual body weight/ideal body weight)] (Yamada et al., 2020). Serum levels of intact FGF23, klotho, fetuin-A, and interleukin-6 were determined using two-site enzyme-linked immunosorbent assays (Elabscience Biotech, Wuhan, China).

\section{Abdominal Aortic Calcification Integration Method: Abdominal Aortic Calcification Score}

All patients underwent lateral lumbar X-ray examinations within 1 week of biochemical blood examinations to assess abdominal aortic calcification corresponding to L1 to L4 (Asher et al., 2021). Each patient was scored based on the length of the calcified plaques identified on the anterior and posterior walls of the abdominal aorta, with each segment scored between 0 and 3 points, as follows: 0 points for no calcification; 1 point for calcification less than one-third of the arterial wall length; 2 points for calcification between one-third and two-thirds of the artery wall length; and 3 points if calcification covers more than two-thirds of the arterial wall length. Each lumbar segment is scored separately for both the posterior and anterior walls, resulting in a total score of $0-24$ points. Table 1 shows the demographic and clinical details of our data set.

\section{The $k$-Local Hyperplane Distance Nearest-Neighbor Model}

Vincent and Bengio developed an improved version of the $k$-nearest-neighbor algorithm (KNN), called the $k$-local hyperplane (LH) nearest-neighbor algorithm (HKNN) (Vincent and Bengio, 2002). The purpose of HKNN is to estimate the distance from the test sample in each class to its corresponding $\mathrm{LH}$, which is built using the nearest k samples of the test sample. Suppose there are $C$ classes; HKNN will obtain the predicted results of the test sample by calculating the minimum distance from C LHs. For the $c$-th class, the $c$-th LH is based on the nearest $k$ neighbors of $x$ in the training set, where $x$ belongs to the $c$-th class. The $c$-th hyperplane is expressed as follows:

$$
L H_{k}^{c}=\left\{p^{c} \mid p^{c}=\bar{N}^{c}+\sum_{i=1}^{k} \alpha_{i}^{c} V_{i}^{c}, \alpha_{i}^{c} \in R^{k}, i=1, \ldots, k\right\}
$$

where $\bar{N}^{c}=\frac{1}{k} \sum_{i=1}^{k} N_{i}^{c}$ is the centroid of the $k$ neighbors of $x$ in class $c$; $N_{i}^{c}$ is the $i$ th neighbor of the test sample $x$ in class $c$; and $V_{i}^{c}=N_{i}^{c}-\bar{N}^{c}$. The objective function of the test sample $x$ to the cth $\mathrm{LH}$ is as follows:

$$
\left(L H_{k}^{c}(x)\right)^{2}=\left\|x-\bar{N}^{c}-\sum_{i=1}^{k} \alpha_{i}^{c} V_{i}^{c}\right\|^{2}+\lambda \sum_{i=1}^{k}\left(\alpha_{i}^{c}\right)^{2}
$$

where $\lambda$ is the parameter of regular term. $\alpha^{c}$ can be calculated as:

$$
\left(\left(V^{c}\right)^{T} V^{c}+\lambda I\right) \alpha^{c}=\left(V^{c}\right)^{T}\left(x-\bar{N}^{c}\right)
$$


TABLE 1 | Data set information.

\begin{tabular}{|c|c|c|c|}
\hline No. & Feature & Value & $r^{*}$ \\
\hline 1 & Sex (male/female) & $32 / 27$ & -0.0455 \\
\hline 2 & Age (years) & $55.83 \pm 15.60$ & 0.4010 \\
\hline 3 & Smoking (yes/no) & $1 / 58$ & -0.0847 \\
\hline 4 & $\mathrm{BMI}\left(\mathrm{kg} / \mathrm{m}^{2}\right)$ & $23.56 \pm 3.12$ & 0.1639 \\
\hline 5 & DM (yes/no) & $24 / 35$ & 0.4847 \\
\hline 6 & $\mathrm{Cl}$ (yes/no) & $3 / 56$ & 0.0025 \\
\hline 7 & CHD (yes/no) & $5 / 54$ & 0.0433 \\
\hline 8 & Systolic blood pressure (mmHg) & $155.69 \pm 23.63$ & -0.1150 \\
\hline 9 & Diastolic blood pressure (mmHg) & $88.11 \pm 13.71$ & -0.2043 \\
\hline 10 & Phosphate binder (yes/no) & $36 / 23$ & -0.2141 \\
\hline 11 & Hemoglobin (g/L) & $85.38 \pm 18.12$ & -0.2584 \\
\hline 12 & C-reactive protein (mg/L) & $11.74 \pm 35.61$ & 0.3016 \\
\hline 13 & Serum creatinine $(\mu \mathrm{mol} / \mathrm{L})$ & $785.09 \pm 368.62$ & -0.4252 \\
\hline 14 & Serum glucose (mmol/L) & $5.48 \pm 2.28$ & 0.2608 \\
\hline 15 & Serum calcium (mmol/L) & $2.08 \pm 0.24$ & 0.0520 \\
\hline 16 & Serum phosphorus (mmol/L) & $1.81 \pm 0.38$ & -0.0862 \\
\hline 17 & Total glyceride (mmol/L) & $1.69 \pm 1.08$ & -0.0542 \\
\hline 18 & Total cholesterol (mmol/L) & $4.53 \pm 1.42$ & -0.0466 \\
\hline 19 & Low density lipoprotein-C (mmol/L) & $2.45 \pm 0.96$ & -0.0252 \\
\hline 20 & High density lipoprotein-C (mmol/L) & $0.97 \pm 0.55$ & 0.0866 \\
\hline 21 & $\mathrm{HbA1c}(\%)$ & $5.82 \pm 1.03$ & 0.2151 \\
\hline 22 & Serum albumin (g/L) & $34.31 \pm 6.61$ & -0.1308 \\
\hline 23 & 25-OH Vitamin D3 (ng/ml) & $7.86 \pm 4.55$ & 0.3850 \\
\hline 24 & iPTH (pg/ml) & $274.50 \pm 306.31$ & -0.0225 \\
\hline 25 & GNRI & $96.06 \pm 12.76$ & -0.0078 \\
\hline 26 & FGF23 (pg/ml) & $32.21 \pm 53.02$ & -0.0966 \\
\hline 27 & Klotho (ng/ml) & $2.38 \pm 2.33$ & 0.0443 \\
\hline 28 & Interleukin-6 (pg/ml) & $25.37 \pm 53.69$ & 0.2634 \\
\hline 29 & Fetuin-A (pg/ml) & $\begin{array}{l}3.0320 \mathrm{e}+05 \\
\pm 2.0606 \mathrm{e}+05\end{array}$ & -0.0234 \\
\hline 30 & AACS & $1.95 \pm 1.55$ & 0.2113 \\
\hline 31 & CHADS2 score & $1.93 \pm 1.11$ & 0.4247 \\
\hline 32 & Group (low-risk group/high-risk group) & $25 / 34$ & 0.4619 \\
\hline 33 & CHA2DS2-VASc score & $2.79 \pm 1.50$ & 0.5097 \\
\hline
\end{tabular}

*Denotes each feature correlated with vascular calcification level using Pearson correlation coefficient ( $r$ ).

$B M I$, body mass index; $D M$, diabetes mellitus; $\mathrm{Cl}$, cerebral ischemia; $C H D$, coronary heart disease; HbA1c, glycated hemoglobin; iPTH, intact parathyroid hormone; GNRI, Geriatric Nutritional Risk Index; FGF23, fibroblast growth factor 23; AACS, Abdominal Aortic Calcification Score, CHADS2, congestive heart failure, hypertension, age = 75 years, diabetes mellitus, stroke; CHA2DS2-VASc, congestive heart failure, hypertension, age $\geq 75$ years, diabetes mellitus, stroke or transient ischemic attack vascular disease, age 65-74 years, sex category.

where $\alpha^{c}=\left(\alpha_{1}^{c}, \alpha_{2}^{c}, \ldots, \alpha_{k}^{c}\right)^{T}$ and $V^{c}=\left(V_{1}^{c}, V_{2}^{c}, \ldots, V_{k}^{c}\right)_{d=k}$. The predictive result for test sample $x$ is defined as:

$$
\text { class } c=\min \left\|x-\bar{N}^{c}-\sum_{i=1}^{k} \alpha_{i}^{c} V_{i}^{c}\right\|^{2}, c=1,2, \ldots, C
$$

To further improve the performance of the model, we applied feature mapping and the kernel trick to HKNN to obtain a kernelized HKNN model (KNKNN). Let $x$ map to $f$ by $\phi: \chi \rightarrow F$ and set $\bar{x}=x-\bar{N}^{c}$. Eq. 2 can then be rewritten, as follows:

$$
\left(L H_{k}^{c}(x)\right)^{2}=\left\|\phi(\bar{x})-\sum_{i=1}^{k} \alpha_{i}^{c} \phi\left(V_{i}^{c}\right)\right\|^{2}+\lambda \sum_{i=1}^{k}\left(\alpha_{i}^{c}\right)^{2}
$$

We obtained the differential of Eq. 5 as follows:

$$
\begin{gathered}
\left(\left(\phi\left(V^{c}\right)\right)^{T} \phi\left(V^{c}\right)+\lambda I\right) \alpha^{c}=\left(\phi\left(V^{c}\right)\right)^{T} \phi(\bar{x}) \\
\alpha^{c}=\left(\left(\phi\left(V^{c}\right)\right)^{T} \phi\left(V^{c}\right)+\lambda I\right)^{-1}\left(\phi\left(V^{c}\right)\right)^{T} \phi(\bar{x}) \\
\alpha^{c}=\left(K\left(V^{c}, V^{c}\right)+\lambda I\right)^{-1} K\left(V^{c}, \bar{x}\right)
\end{gathered}
$$

where $K\left(V^{c}, V^{c}\right) \in R^{k=k}$ is a Gram matrix calculated by the radial basis function (RBF), and $K\left(V^{c}, \bar{x}\right) \in R^{k=1}$ is a vector. The RBF is defined as:

$$
K\left(x_{i}, x_{j}\right)=\exp \left(-\gamma\left\|x_{i}-x_{j}\right\|^{2}\right)
$$

where $\gamma$ is the Gaussian kernel bandwidth.

To avoid overfitting of the model, KHKNN employed two strategies: (1) For test sample, KHKNN separately constructs a local hyperplane for each category by linear representation of neighborhood samples. It can alleviate the parameter (number) sensitivity of neighbors. (2) When constructing the hyperplane, we added the regular term (L2) of the coefficient. The schematic diagram of KNKNN is shown in Figure 1.

\section{RESULTS}

\section{Measurements}

In this study, accuracy (ACC), sensitivity (SN), specificity (SP), positive predictive value (PE), negative predictive value (NPV), a weighted average of the $\mathrm{PE}$ and sensitivity $\left(\mathrm{F}_{\text {score }}\right)$, and Matthews correlation coefficient (MCC) were calculated as follows:

$$
\begin{gathered}
A C C=\frac{T P+T N}{T P+F P+T N+F N} \\
S N=\frac{T P}{T P+F N} \\
S p e c=\frac{T N}{T N+F P} \\
P E=\frac{T P}{T P+F P}
\end{gathered}
$$

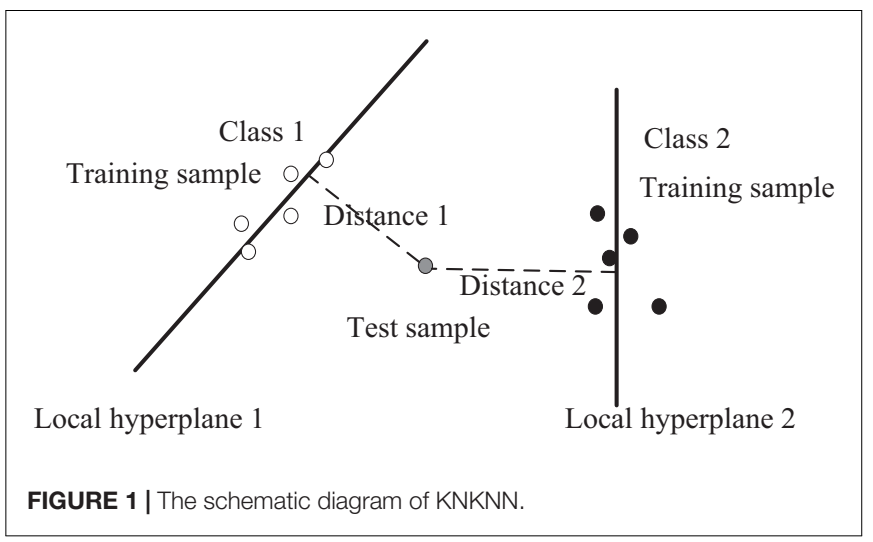




$$
\begin{gathered}
N P V=\frac{T N}{T N+F N} \\
F_{\text {score }}=2 \times \frac{S N \times P E}{S N+P E} \\
M C C=\frac{T P \times T N-F P \times F N}{\sqrt{(T P+F N) \times(T N+F P) \times(T P+F P) \times(T N+F N)}}
\end{gathered}
$$

where $T P, T N, F N$, and $F P$ are the number of true positive, true negative, false negative, and false positive results, respectively. The high-risk patients are positive samples, whereas the low-risk patients are negative samples.

\section{The Optimal Parameters}

In our model, three parameters $(k, \gamma$, and $\lambda)$ must be selected. To make the model robust, we set $\lambda$ as 1 . The optimal parameters of $k$ and $\gamma$ were selected using a grid search. The value of $k$ ranges from 2 to 8 (maximum number of negative samples), with a step of 1 . The value of $\gamma$ ranges from $2^{-5}$ to $2^{5}$ with a step of $2^{1}$. The results are shown in Figure 2, which shows the predictive performance of the model using different parameters. When $k$ and $y$ are 4 and $2^{-3}$, respectively, the best MCC $(0.5393)$ value was obtained.

\section{Comparison of Performance Between Our Method and Other Existing Methods}

We compared the performance of several traditional classifiers with our method using the obtained dataset. The results are shown in Table 2 . The compared classifiers included artificial neural network with backpropagation (ANN-BP), support vector machines (SVM), Takagi-Sugeno-Kang fuzzy system (TSK-FS), KNN, HKNN, and KHKNN. For small samples, KNN (ACC: 86.67\%), HKNN (ACC: 85.00\%), and TSK-FS (ACC: 78.33\%) achieved good results. Our method (KHKNN) achieved the best MCC (0.5393) and ACC (89.67\%) on small dataset. KHKNN was found to have an SN of $94.00 \%$, with Spec reaching $60.00 \%$. Our method achieved a relatively balanced performance for the recognition of both positive and negative samples.

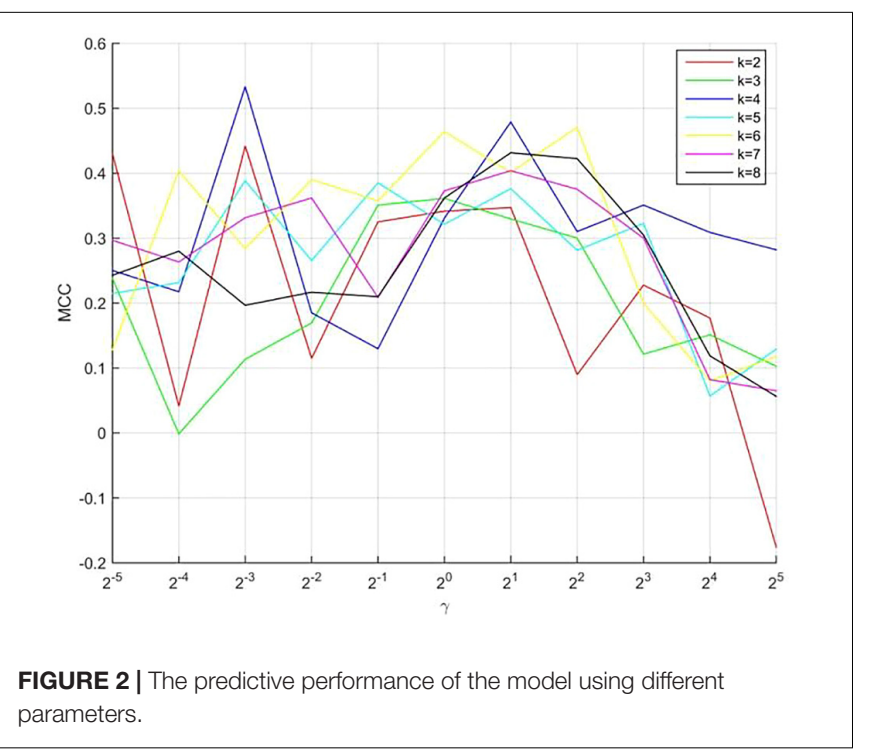

\section{DISCUSSION}

Stroke is one of the most serious complications among patients with CKD, leading to brain dysfunction and even death. Over the past 10 years, scholars have conducted a large number of mechanistic studies and epidemiological investigations exploring the kidney-brain interaction. The results of these studies have indicated that the kidney and brain have similar anatomical and functional characteristics. For example, both organs feature an arterial system that automatically adjusts perfusion pressure to ensure a continuous and relatively stable blood flow. In patients with $\mathrm{CKD}$, cerebrovascular sclerosis occurs due to calcifications that form in the arterial system, disrupting the autoregulation function and allowing cerebrovascular events to occur (Lau et al., 2017).

The risk of stroke in patients with CKD is much higher than that in patients without CKD (Chen et al., 2012), and the stroke risk increases further as renal functional defects progress to ESRD. In recent years, in addition to traditional risk factors, such as hypertension, diabetes, and dyslipidemia, the influence of non-traditional risk factors on the occurrence of cerebrovascular calcification in patients with CKD has gained increasing attention, including inflammation, malnutrition, and the FGF23/klotho axis. The results of previous studies performed at our center have indicated that abnormal FGF23, klotho, and fetuin-A levels and malnutrition represent risk factors for abdominal aortic calcification in patients with ESRD (Maraj et al., 2018). FGF23 has been to play an important role in phosphate regulation. Klotho is the receptor protein for FGF23, which participates in regulating bone, calcium, and phosphorus metabolism; protecting the integrity of blood vessels; and inhibiting vascular calcification through the formation of FGF23klotho complexes. The FGF23/klotho axis is a key participant in CKD-MBD and is closely related to vascular calcification and cerebrovascular diseases (Moldovan et al., 2014). Relevant studies have shown that an elevated FGF23 level is a risk factor for ischemia and hemorrhagic stroke in patients with CKD (Wright et al., 2014).

TABLE 2 | Comparison of performance between our method and other existing methods using the PDB1075 data set (Jackknife test evaluation).

\begin{tabular}{lccccccc}
\hline Methods & MCC & ACC (\%) & SN (\%) & Spec (\%) & PE (\%) & NPV (\%) & F $_{\text {score }}$ \\
\hline ANN-BP & 0.3521 & 77.58 & 82.18 & $\mathbf{6 0 . 0 0}$ & 92.32 & 40.00 & 0.8541 \\
SVM & NaN & 86.52 & 100 & 0 & 86.52 & NaN & 0.9272 \\
TSK-FS & 0.3803 & 78.33 & 82.91 & $\mathbf{6 0 . 0 0}$ & 92.00 & 43.33 & 0.8612 \\
KNN & 0.3697 & 86.67 & $\mathbf{9 4 . 1 8}$ & 40.00 & 90.73 & 50.00 & 0.9237 \\
HKNN & 0.4865 & 85.00 & 90.55 & 50.00 & 91.92 & $\mathbf{6 6 . 6 7}$ & 0.9094 \\
KHKNN & $\mathbf{0 . 5 3 9 3}$ & $\mathbf{8 9 . 6 7}$ & 94.00 & $\mathbf{6 0 . 0 0}$ & $\mathbf{9 4 . 5 2}$ & 63.33 & $\mathbf{0 . 9 4 0 7}$
\end{tabular}

MCC, Matthews correlation coefficient; ACC, accuracy; SN, sensitivity; Spec, specificity; PE, positive predictive value; NPV, negative predictive value; $F_{\text {score, }}$ weighted average of the PE and sensitivity; ANN-BP, artificial neural network with backpropagation; SVM, support vector machines; TSK-FS, Takagi-SugenoKang fuzzy system; KNN, k-nearest-neighbor; HKNN, k-local hyperplane distance nearest-neighbor algorithm; KHKNN, kernelized HKNN; NaN, not a number. The best results in each column are in bold faces. 
The CHADS2 and CHA2DS2-VASc scores are considered to be effective evaluation tools for predicting the risk of ischemic stroke in patients with CKD. Among patients with a high risk of ischemic cerebrovascular accidents, the administration of secondary prevention agents, such as anticoagulation and antithrombosis factors, when indicated by the cerebral ischemia score warning system, has been shown to greatly reduce the incidence of stroke and improve prognosis (Toyoda et al., 2014). The results of this study revealed a correlation between the CHADS2 and CHA2DS2-VASc scores in patients with ESRD and vascular calcification, which indirectly suggests the existence of an important relationship between vascular calcification and ischemic stroke. Moreover, when we combined the ischemic stroke scoring tool with traditional stroke risk factors, such as vascular calcification, to predict the risk of CKD stroke, the results were more reasonable, with a stronger scientific basis, than the use of the stroke scoring tool alone to predict risk.

The sample size is very small, so a simple machine learning model is preferred to solve the classification problem. Among them, KNN and SVM are suitable methods. The KNN algorithm is very sensitive to the number of neighboring samples. In the original feature space, the model cannot achieve satisfactory results of prediction. Therefore, we proposed KHKNN on the basis of KNN and HKNN to solve the above two problems. In the results section, KHKNN has obtained good prediction results. KHKNN separately constructs a local hyperplane for each category of test sample. The prediction result is determined by evaluating the distance (minimum) from the test sample to the hyperplane of each category. Therefore, it can alleviate the parameter sensitivity of KNN and avoid overfitting. The prediction result is determined by evaluating the distance (minimum) from the test sample to the hyperplane of each category.

In our study, KHKNN was employed to predict the risk of cerebrovascular disease among patients with ESRD. KHKNN estimates the distance from the test sample in each class to its corresponding LH in a high-dimensional feature space. Unlike $\mathrm{KNN}, \mathrm{KHKNN}$ is not as sensitive to the parameter $k$, and its prediction performance is better than that of the ANN-BP and SVM models for small data sets. Compared with other models (ANN-BP, SVM, TSK-FS, KNN, and HKNN), our model achieved the best MCC (0.5393) and ACC (89.67\%) values, showing that our method has good robustness and may be useful for determining clinical risk in the future.

\section{CONCLUSION}

We proposed a KHKNN method to filter noise samples, improve the generalization ability of the model, and obtain good results. Although our method achieves a relatively balanced performance for the recognition of positive and negative samples, the following disadvantages must be acknowledged. (1) The sample size must be further increased to minimize prediction bias. (2) No detailed analysis was performed to examine the contribution of various patient factors. (3) Although the kernel function was used to map the original space to further improve the performance, the interpretability of the model was affected. Fuzzy systems will be introduced in the future to improve interpretability. At present, artificial intelligence technology has been used for large-scale medical information processing (Jian et al., 2019; Guo et al., 2021; Jiang et al., 2021a,b; Zhang et al., 2021a,b) and bioinformatics (Qian et al., 2021; Zou et al., 2021) on a large scale, with good performance. (4) The k-dimensional tree is employed to speed up the search speed of the nearest neighbor samples. In addition, parallel computing technology also can increase the speed of searching. In the future, we will use artificial intelligence methods to solve additional clinical problems.

\section{DATA AVAILABILITY STATEMENT}

The original contributions presented in the study are included in the article/supplementary material, further inquiries can be directed to the corresponding author/s.

\section{ETHICS STATEMENT}

The studies involving human participants were reviewed and approved by the Human Ethics Committee (Wuxi People's Hospital Ethics Committee, No. KS2019041). The patients/participants provided their written informed consent to participate in this study.

\section{AUTHOR CONTRIBUTIONS}

XL: methodology, data curation, and writing - original draft preparation. XZ: methodology and writing - original draft preparation. WS and XG: methodology and data curation. YD: methodology and software. LW: methodology, supervision, and writing - reviewing and editing. All authors contributed to the article and approved the submitted version.

\section{FUNDING}

This work is supported by a grant from the Top Talent Support Program for young and middle-aged people of Wuxi Health Committee [HB2020008]; the Scientific Research Project of Wuxi Health Committee [MS201927; Z201914]; the Scientific Research Project of Jiangsu Provincial Health Commission [LGY201801]; Jiangsu Province “333" project [BRA2020142]; Medical and Public Health Project of Wuxi Sci-Tech Development Fund [WX18 II AN047]; and Maternal and Child Health Research Project of Jiangsu Province [F202033].

\section{ACKNOWLEDGMENTS}

We thank the Department of Nephrology of Wuxi People's Hospital for collecting data in this study. 


\section{REFERENCES}

Allen, C. L., and Bayraktutan, U. (2009). Oxidative stress and its role in the pathogenesis of ischaemic stroke. Int. J. Stroke. 4, 461-470. doi: 10.1111/j.17474949.2009.00387.x

Asher, E., Abu-Much, A., Bragazzi, N. L., Younis, A., Younis, A., Masalha, E., et al. (2021). CHADS2 and CHA2DS2-VASc scores as predictors of platelet reactivity in acute coronary syndrome. J. Cardiol. 77, 375-379. doi: 10.1016/j.jjcc.2020. 09.010

Chen, Y. C., Su, Y. C., Lee, C. C., Huang, Y. S., and Hwang, S. J. (2012). Chronic kidney disease itself is a causal risk factor for stroke beyond traditional cardiovascular risk factors: a Nationwide Cohort Study in Taiwan. PLoS One 7:e36332. doi: 10.1371/journal.pone.0036332

de Bie, M. K., Buiten, M. S., Rotmans, J. I., Hogenbirk, M., Schalij, M. J., Rabelink, T. J., et al. (2017). Abdominal aortic calcification on a plain X-Ray and the relation with significant coronary artery disease in asymptomatic chronic dialysis patients. BMC Nephrol. 18:82. doi: 10.1186/s12882-0170480-2

Guo, X., Zhou, W., Shi, B., Wang, X., Du, A., Ding, Y., et al. (2021). An efficient multiple kernel support vector regression model for assessing dry weight of hemodialysis patients. Curr. Bioinform. 16, 284-293. doi: 10.2174/ 1574893615999200614172536

Hsu, P. C., Lee, W. H., Chen, S. C., Tsai, Y. C., Chen, Y. C., Chu, C. Y., et al. (2020). Using CHADS2 and CHA2DS2-VASc Scores for mortality prediction in patients with chronic kidney disease. Sci. Rep. 10:18942. doi: 10.1038/s41598020-76098-y

Jha, V., Garcia-Garcia, G., Iseki, K., Naicker, S., Plattner, B., Saran, R., et al. (2013). Chronic kidney disease: global dimension and perspectives. Lancet 382, 260-272. doi: 10.1016/S0140-6736(13)60687-X

Jian, Y., Zhao, K., Xia, K., Xue, J., Xhou, L., Ding, Y., et al. (2019). A novel distributed multitask fuzzy clustering algorithm for automatic $\mathrm{mr}$ brain image segmentation. J. Med. Syst. 43:118. doi: 10.1007/s10916-019-1245-1

Jiang, Y., Gu, X., Wu, D., Hang, W., Xue, J., Qiu, S., et al. (2021a). A novel negativetransfer-resistant fuzzy clustering model with a shared cross-domain transfer latent space and its application to brain CT image segmentation. IEEE ACM Trans. Comput. Biol. Bioinform. 18, 40-52. doi: 10.1109/TCBB.2019.2963873

Jiang, Y., Zhang, Y., Lin, C., Wu, D., and Lin, C.-T. (2021b). EEG-Based Driver Drowsiness Estimation Using an Online Multi-View and Transfer TSK Fuzzy System. IEEE Trans. Intell. Transp. Syst. 22, 1752-1764. doi: 10.1109/TITS. 2020.2973673

Kelly, D., and Rothwell, P. M. (2020). Disentangling the Multiple Links Between Renal Dysfunction and Cerebrovascular Disease. J. Neurol. Neurosurg. Psychiatry 91, 88-97. doi: 10.1136/jnnp-2019-320526

Lau, W. L., Huisa, B. N., and Fisher, M. (2017). The cerebrovascular-chronic kidney disease connection: perspectives and mechanisms. Transl. Stroke Res. 8, 67-76. doi: 10.1007/s12975-016-0499-X

Liu, X. B., Zhang, X. R., Guo, X. Y., Ding, Y., Shan, W., Wang, L., et al. (2021). A self-representation-based fuzzy svm model for predicting vascular calcification of hemodialysis patients. Comput. Math Methods Med. 2021:2464821. doi: $10.1155 / 2021 / 2464821$

Maraj, M., Kuśnierz-Cabala, B., Dumnicka, P., Gala-Błądzińska, A., Gawlik, K., Pawlica-Gosiewska, D., et al. (2018). Malnutrition, inflammation, atherosclerosis syndrome (MIA) and diet recommendations among end-stage renal disease patients treated with maintenance hemodialysis. Nutrients 10:69. doi: 10.3390/nu10010069

Moldovan, D., Moldovan, I., Rusu, C., Kacso, I., Patiu, I. M., and GhermanCaprioara, M. (2014). FGF-23, vascular calcification, and cardiovascular diseases in chronic hemodialysis patients. Int. Urol. Nephrol. 46, 121-128. doi: 10.1007/s11255-013-0422-2

Qian, Y., Meng, H., Lu, W., Liao, Z., Ding, Y., and Wu, H. (2021). Identification of DNA-binding proteins via hypergraph based laplacian support vector machine. Curr. Bioinform. doi: 10.2174/1574893616666210806091922 [Epub ahead of print].

Toyoda, K., and Ninomiya, T. (2014). Stroke and cerebrovascular diseases in patients with chronic kidney disease. Lancet Neurol. 13, 823-833. doi: 10.1016/ S1474-4422(14)70026-2

Toyoda, K., Yasaka, M., Uchiyama, S., Iwade, K., Koretsune, Y., Nagata, K., et al. (2014). CHADS2 and CHA2DS2-VASc scores as bleeding risk indices for patients with atrial fibrillation: the bleeding with antithrombotic therapy study. Hypertens. Res. 37, 463-466. doi: 10.1038/hr.2013.150

Vincent, P., and Bengio, Y. (2002). K-local hyperplane and convex distance nearest neighbor algorithms. Adv. Neural Inf. Process. Syst. 14, 985-992.

Wright, C. B., Dong, C., Stark, M., Silverberg, S., Rundek, T., Elkind, M. S., et al. (2014). Plasma FGF23 and the risk of stroke: the northern manhattan s tudy (NOMAS). Neurology 82, 1700-1706. doi: 10.1212/WNL.000000000000 0410

Wright, C. B., Shah, N. H., Mendez, A. J., DeRosa, J. T., Yoshita, M., Elkind, M. S., et al. (2016). Fibroblast growth factor 23 is associated with subclinical cerebrovascular damage: the northern manhattan study. Stroke 47, 923-928. doi: 10.1161/STROKEAHA.115.012379

Yamada, S., Yamamoto, S., Fukuma, S., Nakano, T., Tsuruya, K., and Inaba, M. (2020). Geriatric nutritional risk index (GNRI) and creatinine index equally predict the risk of mortality in hemodialysis patients: J-DOPPS. Sci. Rep. 10:5756. doi: 10.1038/s41598-020-62720-6

Zhang, Y. P., Jian, Y., Qi, L., Bhuiyan, M. Z. A., and Quian, P. (2021a). Epilepsy diagnosis using multi-view \& multi-medoid entropy-based clustering with privacy protection. ACM Trans. Internet Technol. 21, 1-20. doi: 10.1145/ 3404893

Zhang, Y. P., Wang, S. H., Xia, K. J., Jiang, Y. Z., and Qian, Q. J. (2021b). alzheimer's disease multiclass diagnosis via multimodal neuroimaging embedding feature selection and fusion. Inf. Fusion. 66, 170-183. doi: 10.1016/j.inffus.2020.09. 002

Zou, Y., Wu, H., Guo, X., Peng, L., Ding, Y., Tang, J., et al. (2021). MK-FSVMSVDD: a multiple kernel-based fuzzy SVM model for predicting DNA-binding proteins via support vector data description. Curr. Bioinform. 16, 274-283. doi: $10.2174 / 1574893615999200607173829$

Conflict of Interest: The authors declare that the research was conducted in the absence of any commercial or financial relationships that could be construed as a potential conflict of interest.

Publisher's Note: All claims expressed in this article are solely those of the authors and do not necessarily represent those of their affiliated organizations, or those of the publisher, the editors and the reviewers. Any product that may be evaluated in this article, or claim that may be made by its manufacturer, is not guaranteed or endorsed by the publisher.

Copyright (c) 2021 Liu, Zhang, Zhang, Ding, Shan, Huang, Wang and Guo. This is an open-access article distributed under the terms of the Creative Commons Attribution License (CC BY). The use, distribution or reproduction in other forums is permitted, provided the original author(s) and the copyright owner(s) are credited and that the original publication in this journal is cited, in accordance with accepted academic practice. No use, distribution or reproduction is permitted which does not comply with these terms. 